\title{
Boundary resistance in magnetic multilayers
}

\author{
B P Vodopyanov ${ }^{1}$ and L R Tagirov ${ }^{1,2} \S$ \\ ${ }^{1}$ Kazan Physical-Technical Institute of RAS, 420029 Kazan, Russia \\ ${ }^{2}$ Kazan State University, 420008 Kazan, Russia
}

\begin{abstract}
Quasiclassical boundary conditions for electrochemical potentials at the interface between diffusive ferromagnetic and non-magnetic metals are derived for the first time. An expression for the boundary resistance accurately accounts for the momentum conservation law as well as essential gradients of the chemical potentials. Conditions are established at which spin-asymmetry of the boundary resistance has positive or negative sign. Dependence of the spin asymmetry and the absolute value of the boundary resistance on the exchange splitting of the conduction band opens up new possibility to estimate spin polarization of the conduction band of ferromagnetic metals. Consistency of the theory is checked on existing experimental data.

PACS numbers: $74.50 .+\mathrm{r}, 74.80 . \mathrm{Dm}, 74.62 .-\mathrm{c}$
\end{abstract}

Submitted to: Published in J. Phys.: Condens. Matter. 18, 1545-1552 (2006)

$\S$ To whom correspondence should be addressed (Lenar.Tagirov@ksu.ru) 


\section{Introduction}

Discovery of giant magnetoresistance (MR) in magnetic multilayers [1, 2], which consist of alternating ferromagnetic metal (F) and normal metal (N) layers, stimulated intensive studies of spin-polarized transport through layered magnetic structures [3, 4, 5]. In a paper [6] the semiclassical theory of MR in magnetic multilayers has been developed for geometry, in which the electric current is perpendicular to the layers (CPP geometry). It has been shown that when thickness of the metals in a stack is small compared with the spin diffusion length, the magnetoresistance of multilayers can be calculated within the two-current series-resistor model [7, 8, 9]. In this case $\mathrm{MR}$ is expressed via the resistance of the $\mathrm{F} / \mathrm{N}$ interface (boundary resistance), $r_{\uparrow(\downarrow)}=2\left[1 \mp \gamma_{F / N}\right] R_{F / N}^{*}$, and the bulk specific resistance of the ferromagnetic layer, $\rho_{\uparrow(\downarrow)}=2\left[1 \mp \beta_{F}\right] \rho_{F}^{*}[\underline{6}$. In these expressions $\gamma_{F / N}$ and $\beta_{F}$ are parameters of spin asymmetry of the boundary and bulk scattering resistances. It follows from works [ $[6$, 10] that their linear combination, $\beta_{F 1} \rho_{F 1}^{*} t_{F 1}+\gamma_{1} R_{F / N}^{*}$, determines sign of MR in layered $[F 1 / N / F 2 / N] \times n$ structures $\left(t_{F}\right.$ is the thickness of the ferromagnetic layers). In works [11, 12 positive (inverse) MR due to negative value of $\gamma_{F / N}$ in $(F 1 / C r / F 2 / C r)$ multilayers has been observed for the first time.

Theoretical calculations of the boundary resistance [13, 14, 15] established the strong influence of the spin-dependent band structure of ferromagnetic metals on magnetoresistance. Using the approach of Ref. [13] and transmission probabilities through the $\mathrm{F} / \mathrm{N}$ interface, calculated earlier from first principles [16], Stiles and Penn 14. obtained from numerical calculations a negative value of $\gamma_{F / N}$ for the $\mathrm{Fe} / \mathrm{Cr}$ interface, and a positive $\gamma_{F / N}$ for iron-group ferromagnet - noble metal interfaces. However, conditions on the parameters of contacting materials resulting in negative or positive values of spin-asymmetry of the boundary resistance were not discussed in the above papers.

In this paper we derive for the first time quasiclassical boundary conditions for electrochemical potentials of diffusive ferromagnetic and normal metals, which can be used for solution of a wide class of problems in spintronics. Our expression for the boundary resistance accurately accounts for the momentum conservation law as well as essential gradients of the chemical potentials. We establish conditions on parameters of the contacting metals, at which spin asymmetry of the boundary resistance has a positive or negative sign. Dependence of the spin asymmetry and the boundary resistance on the exchange splitting of the conduction band offers one more way to estimate spin polarization of conduction band of ferromagnetic metals. We give an example of such an estimation.

\section{Boundary conditions for electrochemical potentials}

We derive boundary conditions for electrochemical potentials of diffusive metals using the quasiclassical Green functions technique. The "diffusive ferromagnetic metal" is 
a metal in which spin splitting of the conduction band is small compared with the momentum relaxation rate for conduction electrons. Let us consider that axis $x$ is perpendicular to the $F / N$ boundary, and neglect reversal of the electron spin upon transmission through the interface. Then, for each of the metals equations for the Green functions $g_{\alpha}(\mathbf{n}, x, \rho, t)$ read [17]:

$$
\begin{aligned}
& v_{x, \alpha} \frac{\partial g_{a, \alpha}}{\partial x}+\mathbf{v}_{\|} \frac{\partial g_{s, \alpha}}{\partial \rho}+\frac{1}{\tau_{\alpha}}\left(g_{s, \alpha}-\bar{g}_{s, \alpha}\right)=0, \\
& v_{x, \alpha} \frac{\partial g_{s, \alpha}}{\partial x}+\mathbf{v}_{\|} \frac{\partial g_{a, \alpha}}{\partial \rho}+\frac{g_{a, \alpha}}{\tau_{\alpha}}=0 .
\end{aligned}
$$

Here $\mathbf{n}=\mathbf{p}_{x, \alpha} /\left|\mathbf{p}_{\alpha}\right| ; g_{s(a), \alpha}=1 / 2\left[g_{\alpha}\left(n_{x}, x, \rho, t\right) \pm g_{\alpha}\left(-n_{x}, x, \rho, t\right)\right]$ is the single-particle quasiclassical Green function symmetric (antisymmetric) with respect to a projection of the Fermi momentum $\mathbf{p}_{x, \alpha}$ on the axis $x ; v_{x}$ is a projection of the Fermi velocity on the axis $x ; \alpha=(\uparrow, \downarrow)$ is a spin index, and $\rho=(y, z)$ is a coordinate in a plane of the contact. The bar above $g_{s, \alpha}$ means integration over the solid angle: $\bar{g}_{s, \alpha}=\oint d \Omega / 2 \pi g_{s, \alpha}$.

The boundary conditions to Eqs. (11) and (2) are as follows [17]:

$$
\begin{aligned}
& g_{a, \alpha}^{F}(0)=g_{a, \alpha}^{N}(0)=\left\{\begin{array}{l}
g_{a, \alpha}(0), \quad p_{\|}<p_{\alpha}^{F}, p^{N} \\
0, \quad \min \left(p_{\alpha}^{F}, p^{N}\right)<p_{\|}
\end{array},\right. \\
& 2 R_{\alpha} g_{a, \alpha}(0)=D_{\alpha}\left(g_{s, \alpha}^{F}(0)-g_{s, \alpha}^{N}(0)\right) \text {. }
\end{aligned}
$$

In the equations (3) and (44) $p_{\alpha}^{F}$ and $p^{N}$ are the Fermi momenta in ferromagnetic and normal metals, respectively; $p_{\|}$is a projection of a momentum on the plane of the contact; $D_{\alpha}$ and $R_{\alpha}=1-D_{\alpha}$ are the spin-dependent, quantum-mechanical transmission and reflection coefficients. Boundary conditions (3), (44) obey the specular reflection law:

$$
p_{\|}=p_{\downarrow}^{F} \sin \theta_{\downarrow}=p_{\uparrow}^{F} \sin \theta_{\uparrow}=p^{N} \sin \theta_{N} .
$$

The angles $\theta$ in (15) are measured from the axis $x$, a range of variation for the biggest one is $[0, \pi / 2]$. The quasiclassical equations (11) and (2), and the boundary conditions (3) and (41) are formulated for a single electron trajectory determined by the angles $\varphi$ and $\theta$.

Upon solution of the system of equations (11) and (2) we shall consider that the ferromagnet is located to the left of the boundary $x=0$, and the normal metal - to the right $(x>0)$, and that the functions $g_{s, \alpha}$ are homogeneous in the plane of the contact. Then, the system of equations (11) and (2) can be solved in the form of integral equations for the functions $g_{a, \alpha}$ and $g_{s, \alpha}$ in the energy representation, $g_{s, \alpha}(\varepsilon)=2 \tanh (\varepsilon / 2 T)+f_{s, \alpha}(\varepsilon)$ :

$$
\begin{aligned}
& f_{s, \alpha}^{N}(x)=g_{a, \alpha}^{N}(x)+\frac{1}{l_{x, \alpha}} \int_{x}^{\infty} d \xi e^{\frac{x-\xi}{l_{x, \alpha}}} \bar{f}_{s, \alpha}^{N}(\xi), \\
& f_{s, \alpha}^{F}(x)=-g_{a, \alpha}^{F}(x)+\frac{1}{l_{x, \alpha}} \int_{-\infty}^{x} d \xi e^{\frac{\xi-x}{l_{x, \alpha}}} \bar{f}_{s, \alpha}^{F}(\xi) .
\end{aligned}
$$


In a dirty metal the solid-angle averaged function $\bar{f}_{s, \alpha}(\xi)$ obeys the diffusion equation with a decay length which is much longer than the mean free path $l_{\alpha}$. Then, we expand $\bar{f}_{s, \alpha}^{F(N)}(\xi)$ in the r.h.s. of Eqs. (6) and (7) near point $x$ and take out from the integrals independent on $\xi$ terms. Substituting the resulting expansions into the boundary condition (4) we find:

$$
2 g_{a, \alpha}(0)=D_{\alpha}\left[\left(1-l_{x, \alpha}^{F} \frac{d}{d x}\right) \bar{f}_{s, \alpha}^{F}(x)-\left(1+l_{x, \alpha}^{N} \frac{d}{d x}\right) \bar{f}_{s, \alpha}^{N}(x)\right]_{x=0} .
$$

To formulate boundary conditions for the functions $\bar{f}_{s, \alpha}^{F(N)}$ (which are, in fact, chemical potentials - see below) we use a matching procedure proposed in Ref. [18. From equation (11) it follows that for distances of the order of $l_{x, \alpha}$ from the interface

$$
\overline{l_{x, \alpha} \frac{d g_{a, \alpha}}{d x}}=0 \text {. }
$$

Hence,

$$
\overline{l_{x, \alpha} g_{a, \alpha}}=C=\text { const }
$$

in each of the metals. Now we calculate, for example, $C^{F}$ using the expression (8) for $g_{a, \alpha}(x=0)$. Then, we calculate $C^{F}$ far from the interface using an approximate expression for $g_{a, \alpha}^{F}(x)$,

$$
g_{a, \alpha}^{F}(x)=-l_{x, \alpha}^{F} \frac{d \bar{f}_{s, \alpha}^{F}(x)}{d x},
$$

which follows from equation (2) after expansion of $g_{a, \alpha}^{F}$ on Legendre polynomials. Equating values of the constant $C^{F}$ calculated in the two ways, and applying relationship between the averaged Green function and the electrochemical potential, $\bar{f}_{s, \alpha}=(2 / \pi) \mu_{\alpha}$, we receive the boundary condition for the electrochemical potentials at the interface $x=0$ :

$$
l_{\alpha}^{F} \frac{d \mu_{\alpha}^{F}(0)}{d x}=\delta_{\alpha}\left(\mu_{\alpha}^{N}(0)-\mu_{\alpha}^{F}(0)\right)
$$

where

$$
\begin{aligned}
& \delta_{\alpha}=\frac{\delta_{1, \alpha}}{1-\delta_{2, \alpha}}, \quad \delta_{1, \alpha}=\frac{3}{2} \int \frac{d \Omega_{F, \alpha}}{2 \pi} \cos \left(\theta_{F, \alpha}\right) D_{\alpha}, \\
& \delta_{2, \alpha}=\frac{3}{2} \int \frac{d \Omega_{F, \alpha}}{2 \pi}\left[x+\left(\frac{p_{\alpha}^{F}}{p^{N}}\right)^{2} \cos \left(\theta_{N}\right)\right] x D_{\alpha}, \\
& x=\cos \left(\theta_{F, \alpha}\right), \quad d \Omega_{F, \alpha}=\sin \left(\theta_{F, \alpha}\right) d \theta_{F, \alpha} d \varphi .
\end{aligned}
$$

The limits of angular integration must satisfy specular reflection conditions at the interface, Eq. (5). When deriving Eq. (12) we have used conservation of the current density at the interface, which follows from Eq. (3),

$$
j_{\alpha}^{F}(0)=\frac{\sigma_{\alpha}^{F}}{e} \frac{d \mu_{\alpha}^{F}(0)}{d x}=\frac{\sigma_{\alpha}^{N}}{e} \frac{d \mu_{\alpha}^{N}(0)}{d x}=j_{\alpha}^{N}(0),
$$

where $\sigma_{\alpha}^{F}$ and $\sigma_{\alpha}^{N}$ are the bulk, spin-channel conductivities of the metals:

$$
\sigma_{\alpha}^{F(N)}=\frac{e^{2}\left(p_{\alpha}^{F(N)}\right)^{2} l_{\alpha}^{F(N)}}{6 \pi^{2}}
$$


Equation (14) is actually the second, complementary to Eq. (12), boundary condition for the semiclassical description of the spin-polarized transport in magnetic multilayers in terms of electrochemical potential.

\section{Resistance of the interface}

The derivative from the electrochemical potential in Eq. (12) can be expressed in terms of the density of current (14), and we find the spin-dependent resistance of the interface $r_{\alpha}:$

$$
\begin{aligned}
& \mu_{\alpha}^{N}(0)-\mu_{\alpha}^{F}(0)=e r_{\alpha} j_{\alpha}, \\
& r_{\alpha}=\frac{6 \pi^{2}}{e^{2}\left(p_{\alpha}^{F}\right)^{2} A} \frac{1-\delta_{2, \alpha}}{\delta_{1, \alpha}}
\end{aligned}
$$

where $A$ is the area of the contact. It follows from equation (17) that, in the quasiclassical approach at specular reflection from the interface, the boundary resistance between ferromagnetic and normal metals is determined only by the Fermi momenta of the contacting metals and coefficient of transmission through the interface.

Experimental data are given for the spin asymmetry of boundary resistance, $\gamma_{F / N}$, and for the renormalized resistance of the interface, $A R_{F / N}^{*}$, determined as follows:

$$
\gamma_{F / N}=\frac{r_{\downarrow}-r_{\uparrow}}{r_{\uparrow}+r_{\downarrow}}, \quad A R_{F / N}^{*}=\frac{A}{4}\left(r_{\downarrow}+r_{\uparrow}\right) .
$$

To calculate dependence of $\gamma_{F / N}$ and $A R_{F / N}^{*}$ on the Fermi momentum of the nonmagnetic metal for various values of the ferromagnet conduction band polarization we have used the Fermi-momentum-mismatch model for the transmission coefficient: $D_{\alpha}=4 v_{x, \alpha}^{N} v_{x, \alpha}^{F} /\left[\left(v_{x, \alpha}^{N}\right)^{2}+\left(v_{x, \alpha}^{F}\right)^{2}\right]$. Results are presented in Figures $1\left(p_{\uparrow}^{F}>p_{\downarrow}^{F}>p^{N}\right)$, $2\left(p_{\uparrow}^{F}>p^{N}>p_{\downarrow}^{F}\right)$ and $3\left(p^{N}>p_{\uparrow}^{F}>p_{\downarrow}^{F}\right)$. From our calculations it follows that for a non-magnetic metal with a low density of conduction electrons (small value of the Fermi momentum $p^{N}$ ) the spin asymmetry of the boundary resistance $\gamma_{F / N}$ is always negative (Fig. 1). On the contrary, for a non-magnetic metal with a high density of conduction electrons the spin asymmetry of the boundary resistance is always positive (Fig. 3). In an intermediate situation $\gamma_{F / N}$ can be negative as well as positive (see Fig. 2 ). To attain the maximum amplitude of negative magnetoresistance in $F / N$ multilayers the spin asymmetry of the boundary resistance $\gamma_{F / N}$ and the asymmetry of the bulk resistance $\beta_{F}$ should be both positive and close to unity. According to our calculations, the Fermi momentum of the non-magnetic metal should be as far as possible close to the Fermi momentum of the majority subband of ferromagnetic metal (Fig. 2, $p^{N} / p_{\uparrow}^{F} \rightarrow 1.0$ and Fig. 3, $p_{\uparrow}^{F} / p^{N} \rightarrow 1.0$ ). The spin asymmetry of the bulk resistance can be adjusted by the type and concentration of impurities in the ferromagnetic metal [19]. Clearly, similar arguments can be applied to the opposite case of negative values of $\gamma_{F / N}$ and $\beta_{F}$, which will result in positive magnetoresistance in multilayers of alternating ferromagnetic and non-magnetic metals. However, a negative asymmetry of the bulk resistance is met less often [19]. Competition of opposite in a sign asymmetries of boundary and bulk 
resistances can result in negative or positive magnetoresistance depending on the choice of materials and thickness of the ferromagnetic layers [12].

\section{Discussion of experiments}

Experiments on CPP transport in multilayers are very complicated because the resistance of a stack of layers of nanometer thickness is very small (order of $f \Omega$ $\mathrm{m}^{2}$ ). Nevertheless, available experimental data (see reviews [4, 20] and references in them, and also works [12, 21, 22]) allow to test the internal consistency of the theory. Multilayers of the iron-group ferromagnetic metals with noble metals most likely belong to case $3\left(p^{N}>p_{\uparrow}^{F}>p_{\downarrow}^{F}\right)$, and parameter of the spin asymmetry $\gamma_{F / N}$ is positive [4, 12, 20, 21, 22]. For example, $\gamma_{C o / C u} \simeq 0.77$ [4]. Then, intersection of the horizontal dash line $\gamma_{C o / C u} \simeq 0.77$ in the top field of Fig. 3 with the curve $\gamma_{F / N}\left(p^{N}\right)$, corresponding to $\delta=0.6$, gives $p_{\uparrow}^{F} / p^{N} \simeq 0.7$. Accepting $p_{\uparrow}^{F}=1.0 \AA^{-1}$ as a trial value for the Fermi momentum of the majority subband of cobalt we receive $p^{C u} \simeq 1.41 \AA^{-1}$, which fits fairly good the free-electron-model value for copper, $p_{F E M}^{C u} \simeq 1.36 \AA^{-1}[23$. There are data for silver as the non-magnetic spacer: $\gamma_{C o / A g} \simeq 0.85[22$. In a similar way, we obtain from Fig. $3, p^{A g} \simeq 1.22 \AA^{-1}$, which fits well the free-electron-model value $p_{F E M}^{A g} \simeq 1.20 \AA^{-1}$ 23 .

Let us look now at consistency of the theory with boundary resistance data. For the combination $\mathrm{Co} / \mathrm{Cu}, A R_{\mathrm{Co} / \mathrm{Cu}}^{*}(\exp ) \simeq 0.51 f \Omega m^{2}$ [4]. Continuing the vertical dash line for copper in Fig. 3 into the bottom field till intersection with the curve $A R_{F / N}^{*}\left(p^{N}\right)$, corresponding to $\delta=0.6$, we obtain $A R_{C o / C u}^{*}$ (theor) $\simeq 0.74 f \Omega \mathrm{m}^{2}$. A similar procedure gives for silver $A R_{C o / A g}^{*}$ (theor) $\simeq 0.69 f \Omega m^{2}$ (compare with $A R_{C o / A g}^{*}(\exp ) \simeq 0.56 f \Omega m^{2}$ [22, 12]). It is worthy to notice that the theory reproduces fairly good closeness of the boundary resistances of the $\mathrm{Co} / \mathrm{Cu}$ and $\mathrm{Co} / \mathrm{Ag}$ interfaces.

For $\mathrm{Co} / \mathrm{Cr}$ multilayers the asymmetry of the boundary resistance is negative, $\gamma_{C o / C r} \simeq-0.24$ [12]. This value is admissible for cases 1 (Fig.1, top field) and 2 (Fig. 2, top field). Without details, we conclude that the first case does not match the expected value of the conduction band polarization parameter for Co $\delta \sim 0.6 \pm 0.1$ as well as results in about two orders in magnitude higher boundary resistance. The second case (see dash lines in Fig. 2) results in a Fermi momentum $p^{C r} \sim 0.68 \AA^{-1}$, and in a boundary resistance $A R_{C o / C r}^{*}$ (theor $) \simeq 0.61 f \Omega m^{2}$. The Fermi momentum is satisfactory in the frame of the free electron model [23]. The boundary resistance is close enough to the experimental value $A R_{C o / C r}^{*}(\exp ) \simeq 0.48 f \Omega m^{2}[12$. We expect that better matching of the band structures of cobalt and chromium, both belonging to the iron-group metals, would results in a weaker influence of the real band structure on the boundary resistance.

A discrepancy with experiment in an absolute value of boundary resistance of about 20-45\% seems not catastrophic because of the following reasons. First, the trial choice of $p_{\uparrow}^{F}=1.0 \AA^{-1}$ was not optimized. Second, we used the free $s$-electron model as a background for the theory. One may expect that for an interface between a metal 
with predominantly $d$-electron conduction band (iron group) and an $s$-electron metal $(\mathrm{Cu}, \mathrm{Ag})$, reduced overlapping and symmetry mismatch may noticeably increase the boundary resistance. Third, García and Stoll have shown (Fig. 2 of Ref. [24]) that the interface roughness also increases boundary resistance. This increase is estimated below $20-60 \%$ for different models and magnitudes of the interface roughness, and reasonable differences in the Fermi momenta of contacting metals. The interface roughness is much less important for the case of CPP transport, considered in this paper, than for current-in-plane (CIP) transport [25]. Finally, spin-reversal at the interface opposes the both previously considered processes, decreasing the boundary resistance. The reversal of the electron spin by the spin-orbit interaction is always expected upon refraction of the electron wave or scattering on roughness at the interface between two metals. Quantitative analysis of the competition between the above minor mechanisms of boundary resistance is beyond the scope of the paper. However, it is our expectation that the key quantity, spin asymmetry of the boundary resistance $\gamma_{F / N}$, is only weakly dependent on band structures matching, interface roughness, spin reversal etc. because of considerable cancellations in the dimensionless ratio, Eq. (18).

Our trial evaluations show that the experimental data for the spin asymmetry of boundary resistance and the absolute value of boundary resistance in $\mathrm{Co} / \mathrm{Cu}, \mathrm{Co} / \mathrm{Ag}$ and $\mathrm{Co} / \mathrm{Cr}$ multilayers can be consistently described with the use of the spin polarization parameter for the conduction band of cobalt $\delta \simeq 0.6$. At the level of the experimental accuracy and completeness of the theory the estimated value of $\delta$ is identical to $\delta \simeq 0.57$, which we have estimated [26] from experiments of García et al. [27] on magnetoresistance of cobalt nanocontacts. Experiments on Andreev spectroscopy cite similar values of $\delta$ for cobalt [28]. Thus, the spin asymmetry of the boundary resistance in combination with the absolute value of the boundary resistance can be used for estimations of the spin-polarization parameter $\delta$ of the conduction band of ferromagnetic metals.

\section{Acknowledgments}

The work was supported by EC via the Contract No NMP4-CT-2003-505282, and by RFBR via projects No 03-02-17432 and No 03-02-17656. L R T acknowledges numerous illuminating discussions with Professor Nicolas García.

\section{References}

[1] Baibich M N , Broto J M , Fert A, Nguyen Van Dau F, Petroff F, Etienne P, Creuzet G, Friederich A and Chazelas J 1988 Phys. Rev. Lett. 612472.

[2] Binash G, Grunberg P, Saurenbach F and Zinn W 1989 Phys. Rev. B 394828.

[3] Gijs M A M and Bauer G E W 1997 Adv. Phys. 46, 285.

[4] Bass J and Pratt W P Jr. 1999 J. Magn. Magn. Mater. 200274.

[5] Žutić I, Fabian J and Das Sarma S 2004 Rev. Mod. Phys. 76323.

[6] Valet T and Fert A 1993 Phys. Rev. B 487099.

[7] Zhang S and Levy P M 1991 J. Appl. Phys. 694786.

[8] Bauer G E W 1992 Phys. Rev. Lett. 6916676. 
[9] Lee S F, Pratt W P Jr., Yang Z, Holody P, Loloee R, Schroeder P A and Bass J 1993 J. Magn. Magn. Mater. 118 L1.

[10] Rashba E I 2002 Eur. Phys. J. B29 513.

[11] Vouille C, Fert A, Barthelemy A, Hsu S Y, Loloee R and Schroeder P A 1997 J. Appl. Phys. 81 4573.

[12] Vouille C, Barthelemy A, Mpondo F E, Fert A, Schroeder P A, Hsu S Y, Reilly A and Loloee R 1999 Phys. Rev. B 606710.

[13] Schep K M, van Hoof J B A N, Kelly P J, Bauer G E W and Inglesfield J E 1997 Phys. Rev. B 5610805.

[14] Stiles M D and Penn D R 2000 Phys. Rev. B 613200.

[15] Xia K, Kelly P J, Bauer G E W, Turek I, Kudrnovsky J and Drchal V 2001 Phys. Rev. B 63, 64407.

[16] Stiles M D 1996 J. Appl. Phys. 79, 5805; Phys. Rev. B 5414679.

[17] Zaitsev A V 1984 ZhETF 861742 [1984 Sov. Phys. JETP 59 1015].

[18] Kupriyanov M Yu and Lukichev V F 1988 ZhETF 94139 [1988 Sov. Phys. JETP 67 1163].

[19] Fert A and Campbell I A 1976 J. Phys. F 6, 849.

[20] Bass J and Pratt W P Jr. 2002 Physica B 3211.

[21] Zambano A, Eid K, Loloee R, Pratt W P Jr. and Bass J 2002 J. Magn. Magn. Mater. 25351.

[22] Lee S-F, Yang Q, Holody P, Loloee R, Hetherington J H, Mahmood S, Ikegami B, Vigen K, Henry L L, Schoeder P A, Pratt W P Jr. and Bass J 1995 Phys. Rev. 5215426.

[23] Ashkroft N W and Mermin N D 1976 Solid State Physics (Saunders, Philadelphia) Table 2.1.

[24] García N and Stoll E 1988 Phys. Rev. B 37, 4415.

[25] García N, private communication.

[26] Tagirov L R, Vodopyanov B P and Efetov K B 2002 Phys. Rev. B 65214419.

[27] Tatara G, Zhao Y-W, Muñoz M and García N 1999 Phys. Rev. Lett. 832030.

[28] Soulen R J Jr., Osofsky M S, Nadgorny B, Ambrose T, Broussard P, Byers J, Tanaka C T, Nowack J, Moodera J S, Laprade G, Barry A and Coey M D 1999 J. Appl. Phys. 854589.

\section{Figure captions}

Fig. 1. Dependence of the spin-asymmetry of boundary resistance $\gamma_{F / N}$ (top field) and the renormalized resistance of the interface $A R_{F / N}^{*}$ (bottom field) on the Fermi momentum of the non-magnetic metal for the case $\left(p_{\uparrow}^{F}>p_{\downarrow}^{F}>p^{N}\right)$.

Fig. 2. The same as in Figure 1, but for the case $\left(p_{\uparrow}^{F}>p^{N}>p_{\downarrow}^{F}\right)$.

Fig. 3. The same as in Figure 1, but for the case $\left(p^{N}>p_{\uparrow}^{F}>p_{\downarrow}^{F}\right)$. 


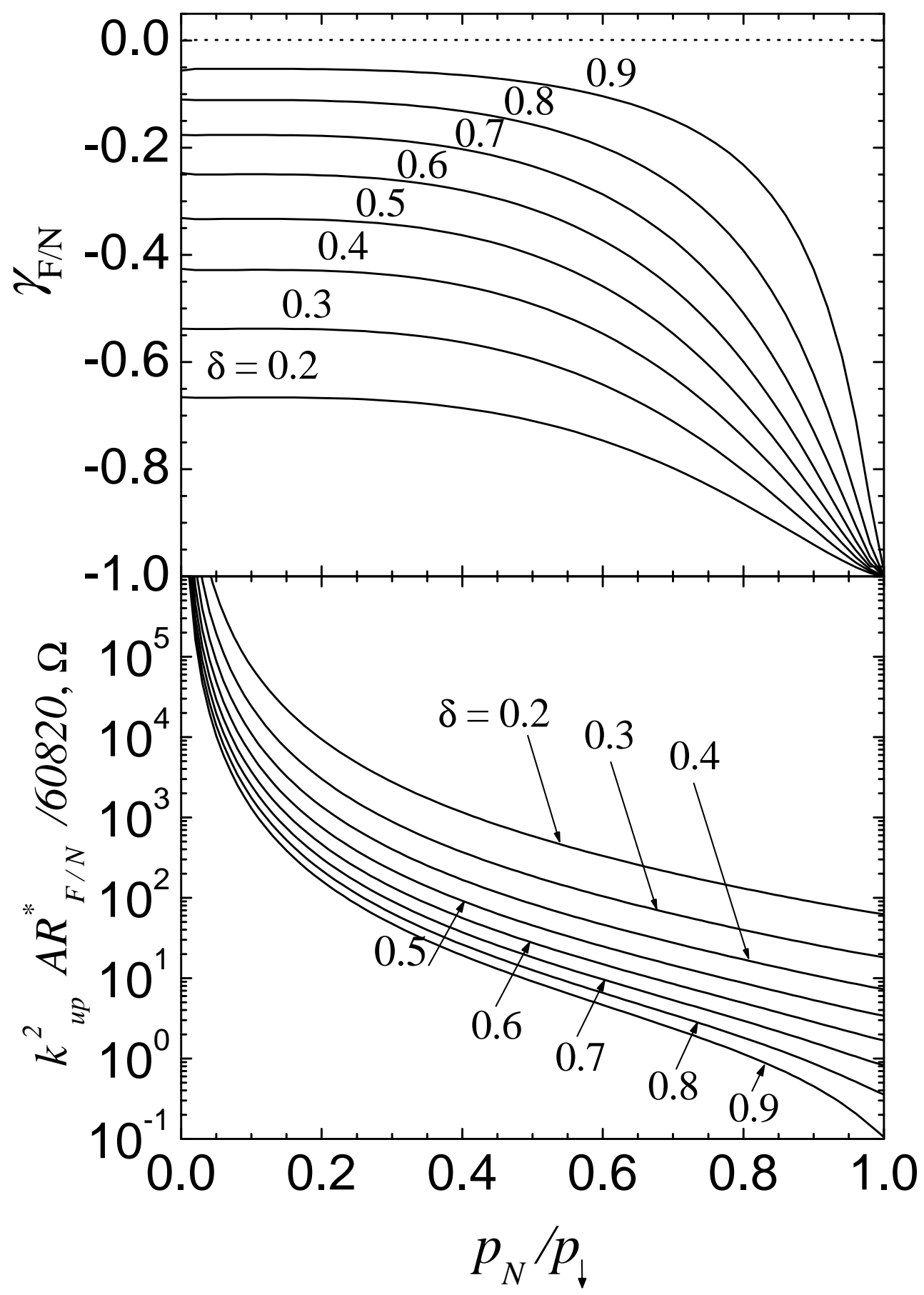

Case 1: $p_{\text {up }}>p_{\text {down }}>p_{N}$ 


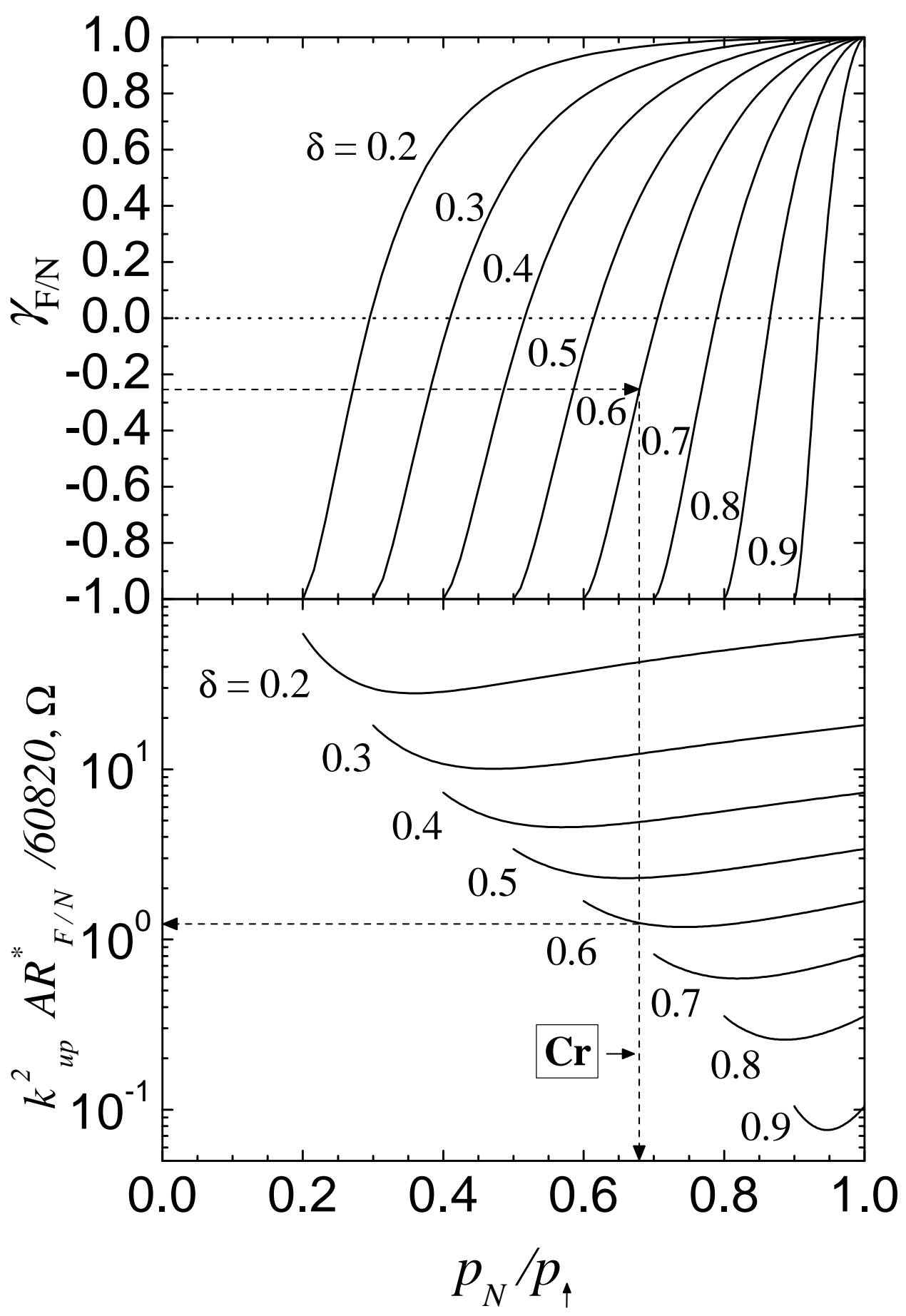

Case 2: $p_{u p}>p_{N}>p_{\text {down }}$ 


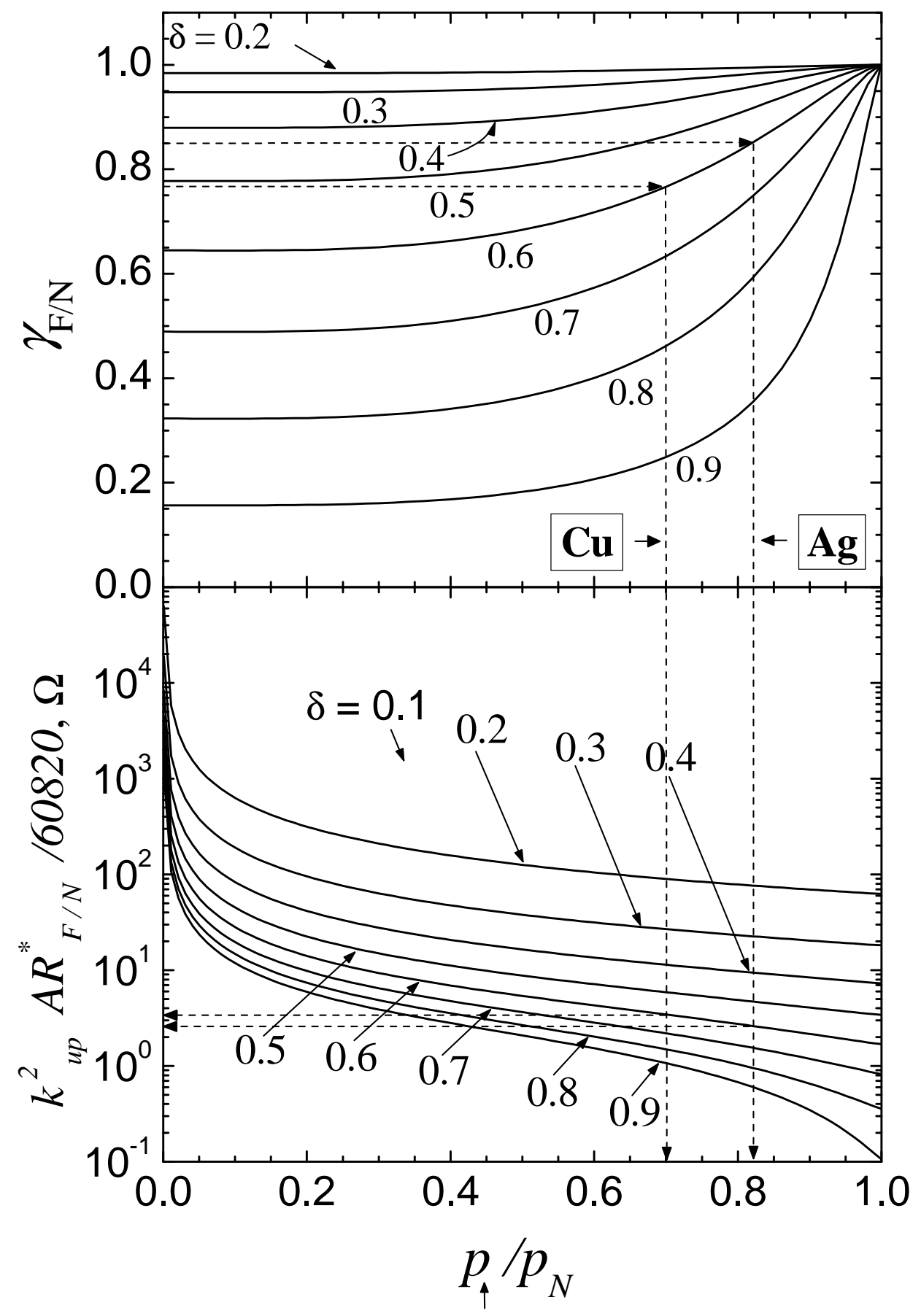

Case 3: $p_{N}>p_{u p}>p_{\text {down }}$ 